\title{
ON THE HIGHER WAHL MAPS
}

\author{
Dedicated to Prof. S. Koizumi on his 70-th birthday
}

By

\section{Akira OHBUCHI}

\section{Introduction}

Let $C$ be a complete non-singular curve defined over $\mathrm{rn}$ algebraically closed field $k$ and let $\mathcal{L}$ be an invertible sheaf of positive degree on $C$. J. Wahl defines a natural map

$$
\Phi_{\mathcal{L}}: \wedge^{2} \Gamma(C, \mathcal{L}) \longrightarrow \Gamma\left(C, \omega_{C} \otimes \mathcal{L}^{\otimes 2}\right)
$$

given by $\Phi_{\mathcal{L}}(s \wedge t)=s(d t)-t(d s)$ where $s, t \in \Gamma(C, \mathcal{L})$ (see [18]). This notation is locally defined and well-defined on $C$. We often call this map a Wahl map. The original study of a Wahl map is the study of $\Phi_{\omega_{C}}$ where $\omega_{C}$ is the canonical sheaf on $C$. This map is very much useful. For example it gives a property which must be satisfied in order that a curve sits on a K3 surface. Precisely if $C$ lies on a K3 surface, then $\Phi_{\omega_{C}}$ is not surjective (see [17]). In [3], we have that if $C$ is a general curve of genus 10 or $\geqq 12$, then $\Phi_{\omega_{C}}$ is surjective. This result gives an answer of Mukai conjecture. Let $\mathcal{L}$ and $\mathscr{M}$ be two invertible sheaves on $C$ and let

$$
\mathscr{R}(\mathcal{L}, \mathscr{M})=\operatorname{ker}[\Gamma(C, \mathcal{L}) \otimes \Gamma(C, \mathscr{M}) \stackrel{\text { cup product }}{\longrightarrow} \Gamma(C, \mathcal{L} \otimes \mathscr{M})] .
$$

In [18], Wahl constructs another Wahl map

$$
\Phi_{\mathcal{L}, \mathscr{M}}: \mathscr{R}(\mathcal{L}, \mathscr{M}) \longrightarrow \Gamma\left(C, \omega_{\mathcal{C}} \otimes \mathcal{L} \otimes \mathscr{M}\right),
$$

(if $\mathcal{L}=\mathscr{M}$, then $\wedge^{2} \Gamma(C, \mathcal{L}) \subset \mathscr{R}(\mathcal{L} \otimes \mathcal{L})$ and $\left.\Phi_{\mathcal{L}, \mathcal{L}}=\Phi_{\mathcal{L}}\right)$. And he proves that if $\operatorname{deg}(\mathcal{L}) \geqq 5 \mathrm{~g}+2$ and $\operatorname{deg}(\mathscr{M}) \geqq 2 \mathrm{~g}+2$, then $\Phi_{\mathcal{L}, \mathscr{M}}$ is surjective, and if $C$ is a non-hyperelliptic curve and $\operatorname{deg}(\mathcal{L}) \geqq 5 \mathrm{~g}+2$, then $\Phi_{\omega_{C}, \mathcal{L}}$ is surjective. These results give several informations about first ordered deformations of a cone over the $C \subset \boldsymbol{P}(\Gamma(C, \mathcal{L})$ ) when $\mathcal{L}$ is a normally generated (very ample) invertible sheaf on $C$ (if $\operatorname{deg}(\mathcal{L}) \geqq 5 \mathrm{~g}+2$ then $\mathcal{L}$ is clearly normally generated (and very ample)). For example we havə that if $\operatorname{deg}(\mathcal{L}) \geqq 5 \mathrm{~g}+2$ then a cone over a non-hyperelliptic curve embedded by $\mathcal{L}$ has only one canonical deformation

Received April 10, 1992. Revised May 17, 1993. 
from the above results (see [17]). $\Phi_{\mathcal{L}}$ also has a geometric meaning. The geometric aspect of a Wahl map is the following. Let $\mathcal{L}$ be a very ample invertible sheaf and $C \hookrightarrow \boldsymbol{P}^{m}$ is an embedding defined by $\mathcal{L}$. Then we can consider a Gaussian map

$$
g: C \longrightarrow \operatorname{Grass}\left(\boldsymbol{P}^{1}, \boldsymbol{P}^{m}\right)
$$

which is given by $g(p)=$ the tangent line of $C$ in $\boldsymbol{P}^{m}$ at $p$ where $\operatorname{Grass}\left(\boldsymbol{P}^{1}, \boldsymbol{P}^{m}\right)$ is a Grassmannian variety of all projective lines in $\boldsymbol{P}^{m}$. Let

$$
\iota: \operatorname{Grass}\left(\boldsymbol{P}^{1}, \boldsymbol{P}^{m}\right) \subset \boldsymbol{P}^{M}
$$

be a Plücker embedding. Then the restriction map

$$
g^{*} \iota^{*}: \Gamma\left(\boldsymbol{P}^{M}, \mathcal{O}_{\boldsymbol{P} M}(1)\right) \longrightarrow \Gamma\left(\boldsymbol{P}^{M}, g^{*} \iota^{*} \mathcal{O}_{\boldsymbol{P}^{M}}(1)\right)
$$

gives the above $\Phi_{\perp}$. Sometimes we call the image $\iota g(C)$ a dual curva of $C$. If $\Phi_{\mathcal{L}}$ is surjective, then the dual curve $\iota g(C)$ is linearly normal and if $\Phi_{\mathcal{L}}$ is injective, then the dual curve $\operatorname{cg}(C)$ is non-degenerate. Therefore the above dual curve $\operatorname{cg}(C)$ is linearly normal if $\operatorname{deg}(\mathcal{L})$ is sufficiently large. In this paper, we want to generalize a Wahl map from the viewpoint of projective geometry. The notion of dual curve is generalized as follows. Let $C \rightarrow \boldsymbol{P}^{m}$ be a birational morphism to its image, let

$$
g_{n}: C \cdots \longrightarrow \operatorname{Grass}\left(\boldsymbol{P}^{n}, \boldsymbol{P}^{m}\right)
$$

be a Gaussian map defined by $g_{n}(p)=$ the osculating tangent $n$-th plaine at $p$ and let

$$
\iota_{n}: \operatorname{Grass}\left(\boldsymbol{P}^{1}, \boldsymbol{P}^{m}\right) \subset \boldsymbol{P}^{M_{n}}
$$

be a Plücker embedding. Then the image $\iota_{n} g_{n}(C)$ is also called a dual curve, and in projective geometry, whether $\iota_{m-1} g_{m-1}(C)$ is linearly normal or not and whether $\iota_{m-1} g_{m-1}(C)$ is non-degenerate or not are very big problems. These conditions are equivalent to surjectivity or injectivity of $g_{m-1}^{*} \ell_{m-1}^{*}$. Let $\mathcal{L}$ be a very ample invertible sheaf on $C$. In section 1 , we define a generalized Wahl map

$$
\Phi_{\mathcal{L}}^{(n)}: \wedge^{n} \Gamma(C, \mathcal{L}) \longrightarrow \Gamma\left(C, \omega_{C}^{\otimes n(n-1) / 2} \otimes \mathcal{L}^{\otimes n}\right)
$$

which is equal to $g_{n}^{*} \iota_{n}^{*}$ if $\operatorname{deg}(\mathcal{L})$ is sufficiently large. Unfortunately we can not give the sufficient conditions for surjectivity or injectivity of $g_{m-1}^{*} \ell_{m-1}^{*}$. But in the sections 2 and 3, we have the following main theorem:

THEOREM. Let $C$ be a non-singular curve of genus $g$ defined over an algebraically closed field $k$ and let $\mathcal{L}$ be an invertible sheaf on $C$. We assume that $\operatorname{char}(k)=0$ or $\operatorname{char}(k)>\operatorname{deg}(\mathcal{L})$. If $\operatorname{deg}(\mathcal{L})>(g-1)\left(2 n^{2}-2 n+3\right)+2\left(n^{2}-1\right)$, then 
$\Phi_{\perp}^{(n)}$ is surjective.

I would like to express my sincerely gratitude to Professor Masaaki Homma for his help and continuous support.

\section{NOTATIONS}

char $(k)$ : The characteristic of a field $k$

$\mathcal{O}_{C}$ : The structure sheaf of a variety $C$

$\omega_{C}$ : The canonical invertible sheaf on a non-singular variety $C$

$f^{*}$ : The pull back defined by a morphism $f$

$\operatorname{deg}(\mathcal{L})$ : The degree of an invertible sheaf $\mathcal{L}$

$\mathcal{O}_{C}(D)$ : The invertible sheaf associated with a divisor $D$

$\Gamma(C, \mathscr{F})$ : The global sections of a sheaf $\mathscr{F}$

$H^{i}(C, \mathscr{F})$ : The $i$-th cohomology group of a sheaf $\mathscr{F}$

$\mathfrak{S}_{n}:$ The symmetric group of degree $n$

$\wedge^{n} V$ : The exterior product of a vector space $V$

$V^{*}:$ The dual space of a vector space $V$

$\sum_{\lambda \in A} V_{\lambda}:$ The direct sum of vector spaces $V_{\lambda}(\lambda \in \Lambda)$

$V \oplus W$ : The direct sum of vector spaces $V$ and $W$

$\boldsymbol{P}(V)$ : The projective space of all 1-dimensional subspaces of $V$

$\operatorname{Grass}\left(\boldsymbol{P}^{n}, \boldsymbol{P}^{m}\right)$ : The Gramann variety of all $n$-plaines in $\boldsymbol{P}^{m}$.

\section{The definition of a higher Wahl map and its basic property}

Let $C$ be a complete non-singular algebraic curve of genus $g$ defined over an algebraically closed field $k$ and let $\mathcal{L}$ be an invertible sheaf on $C$. Throughout of this paper, we assume that $\operatorname{char}(k)=0$ or $\operatorname{char}(k)<\operatorname{deg}(\mathcal{L})$.

Definition 1. Let $V$ be a vector subspace of $\Gamma(C, \mathcal{L})$. We define the $n$ Wahl map

by

$$
\Phi_{V}^{(n)}: \wedge^{n} V \longrightarrow \Gamma\left(C, \omega_{C}^{\otimes n(n-1) / 2} \otimes \mathcal{L}^{\otimes n}\right)
$$

$$
\Phi_{V}^{(n)}\left(s_{1} \wedge \cdots \wedge s_{n}\right)=\left|\begin{array}{ccc}
s_{1} & \cdots & d^{n-1} s_{1} \\
\vdots & & \vdots \\
s_{n} & \cdots & d^{n-1} s_{n}
\end{array}\right|
$$

If $V=\Gamma(C, \mathcal{L})$, we dəfine $\Phi_{\mathcal{L}}^{(n)}$ to be $\Phi_{V}^{(n)}$.

This definition is well-defined. Because if $n=1$, then the proof is found in 
Wahl [18] (see p. 77) and if $n>1$, then the proof is given by the same argument.

Definition 2. Let $V$ be a finite dimensional vector space over $k$. Then for each $x_{1} \wedge \cdots \wedge x_{n}, y_{1} \wedge \cdots \wedge y_{n} \in \wedge{ }^{n} V$, we define

$$
\begin{aligned}
& {\left[x_{1} \wedge \cdots \wedge x_{n}\right] \wedge\left[y_{1} \wedge \cdots \wedge y_{n}\right]} \\
& \qquad \begin{array}{l}
\frac{1}{2}(-1)^{n+1}\left(x_{1} \wedge \cdots \wedge x_{n} \sum_{i=1}^{n}(-1)^{i-1} y_{i} \otimes y_{1} \wedge \cdots \wedge \hat{y}_{i} \wedge \cdots \wedge y_{n}\right. \\
\left.\quad-y_{1} \wedge \cdots \wedge y_{n} \wedge \sum_{i=1}^{n}(-1)^{i-1} x_{i} \otimes x_{1} \wedge \cdots \wedge \hat{x}_{i} \wedge \cdots \wedge x_{n}\right)
\end{array}
\end{aligned}
$$

where $\hat{x}$ means that the term $x$ is omitted.

Clearly this definition is also well-defined. In the above definition,

$$
\left.x_{1} \wedge \cdots \wedge x_{n}\right] \wedge\left[y_{1} \wedge \cdots \wedge y_{n}\right]
$$

is contained in $\wedge^{n+1} V \otimes \wedge^{n-1} V$. According to Definition 1 and Definition 2, we have the following lemma:

LEMMA 1. For every $x_{1}, \cdots, x_{n-1}, y_{1}, \cdots, y_{n-1} \in \Gamma(C, \mathcal{L})$, we have

$$
\begin{aligned}
& \Phi_{\perp}^{(n)} \cap \Phi_{\perp}^{(n-2}\left(\left[x_{1} \wedge \cdots \wedge x_{n-1}\right] \wedge\left[y_{1} \wedge \cdots \wedge y_{n-1}\right]\right) \\
& \quad=\Phi_{\omega_{C}^{(n)}(n)(n-2) / 2}^{(n)} \mathcal{L}^{\otimes n-1}\left(\Phi_{\perp}^{(n-1)}\left(x_{1} \wedge \cdots \wedge x_{n-1}\right) \wedge \Phi_{\perp}^{(n-1)}\left(y_{1} \wedge \cdots \wedge y_{n-1}\right)\right)
\end{aligned}
$$

Proof. By the definition,

$$
\begin{aligned}
& \Phi_{\mathcal{L}}^{(n)} \cap \Phi_{\mathcal{L}}^{(n-2)}\left(\left[x_{1} \wedge \cdots \wedge x_{n-1}\right] \wedge\left[y_{1} \wedge \cdots \wedge y_{n-1}\right]\right) \\
& =\frac{1}{2}(-1)^{n} \Phi_{\mathcal{L}}^{(n)} \cap \Phi_{\mathcal{L}}^{(n-2)}\left(x_{1} \wedge \cdots \wedge x_{n-1} \wedge \sum_{i=1}^{n}(-1)^{i-1} y_{i} \otimes y_{1} \wedge \cdots \wedge \hat{y}_{i} \wedge \cdots \wedge y_{n-1}\right. \\
& \left.-x_{1} \wedge \cdots \wedge x_{n-1} \wedge \sum_{i=1}^{n}(-1)^{i-1} x_{i} \otimes x_{1} \wedge \cdots \wedge \hat{x}_{i} \wedge \cdots \wedge x_{n-1}\right) \\
& =\frac{1}{2}(-1)^{n}\left(\sum_{i=1}^{n-1}(-1)^{i-1}\left|\begin{array}{ccc}
x_{1} & \cdots & d^{n-1} x \\
\vdots & & \vdots \\
x_{n-1} & \cdots & d^{n-1} x_{n-1} \\
y_{i} & \cdots & d^{n-1} y_{i}
\end{array}\right|\left|\begin{array}{ccc}
y_{1} & \cdots & d^{n-3} y_{1} \\
\vdots & \vdots \\
y_{i} & \cdots & d^{n-3} y_{i}< \\
\vdots & \vdots \\
y_{n-1} & \cdots & d^{n-3} y_{n-1}
\end{array}\right|\right. \\
& \left.-\sum_{i=1}^{n-1}(-1)^{i-1}\left|\begin{array}{ccc}
y_{1} & \cdots & d^{n-1} y_{1} \\
\vdots & & \vdots \\
y_{n-1} & \cdots & d^{n-1} y_{n-1} \\
x_{i} & \cdots & d^{n-1} x_{i}
\end{array}\right|\left|\begin{array}{ccc}
x_{1} & \cdots & d^{n-3} x_{1} \\
\vdots & & \vdots \\
x_{i} & \cdots & d^{n-3} x_{i}< \\
\vdots & & \vdots \\
x_{n-1} & \cdots & d^{n-3} x_{n-1}
\end{array}\right|\right)
\end{aligned}
$$


where $><$ means that this low (or column) is omitted. First we calculate the first term of the right-hand side of this equation.

$$
\begin{aligned}
& (-1)^{n} \sum_{i=1}^{n-1}(-1)^{i-1}\left|\begin{array}{ccc}
x_{1} & \cdots & d^{n-1} x_{1} \\
\vdots & & \vdots \\
x_{n-1} & \cdots & d^{n-1} x_{n-1} \\
y_{i} & \cdots & d^{n-1} y_{i}
\end{array}\right|\left|\begin{array}{ccc}
y_{1} & \cdots & d^{n-3} y_{1} \\
\vdots & & \vdots \\
>y_{i} & \cdots & d^{n-3} y_{i}< \\
\vdots & & \vdots \\
y_{n-1} & \cdots & d^{n-1} y_{n-1}
\end{array}\right| \\
& =(-1)^{2 n} \sum_{i=1}^{n-1} \sum_{j=1}^{n}(-1)^{i+j-1}\left(d^{j-1} y_{i}\right) \\
& \left|\begin{array}{ccccc}
x_{1} & \cdots & d^{j-1} x_{1} & \cdots & d^{n-1} x_{1} \\
\vdots & & \vdots & & \\
x_{n-1} & \cdots & d^{j-1} x_{n-1} & \cdots & d^{n-1} x_{n-1}
\end{array}\right|\left|\begin{array}{ccc}
y_{1} & \cdots & d^{n-3} y_{1} \\
\vdots & & \vdots \\
>y_{i} & \cdots & d^{n-3} y_{i}< \\
\vdots & & \vdots \\
y_{n-1} & \cdots & d^{n-3} y_{n-1}
\end{array}\right| \\
& =-\sum_{j=1}^{n}(-1)^{j-1}\left(\sum_{i=1}^{n-1}(-1)^{i-1}\left(d^{j-1} y_{i}\right)\left|\begin{array}{ccc}
y_{1} & \cdots & d^{n-3} y_{1} \\
\vdots & & \vdots \\
>y_{i} & \cdots & d^{n-3} y_{i}< \\
\vdots & & \vdots \\
y_{n-1} & \cdots & d^{n-3} y_{n-1}
\end{array}\right|\right. \\
& \left.\left|\begin{array}{ccccc} 
& & \bigvee & & \\
x_{1} & \cdots & d^{j-1} x_{1} & \cdots & d^{n-1} x_{1} \\
\vdots & & \vdots & & \vdots \\
x_{n-1} & \cdots & d^{j-1} x_{n-1} & \cdots & d^{n-1} x_{n-1}
\end{array}\right|\right) \\
& =-\sum_{j=1}^{n}(-1)^{j-1}\left(\sum_{i=1}^{n-1}\left|\begin{array}{cccc}
0 & y_{1} & \cdots & d^{n-3} y_{1} \\
\vdots & \vdots & & \vdots \\
d^{j-1} y_{i} & y_{i} & \cdots & d^{n-3} y_{i} \\
\vdots & \vdots & & \vdots \\
0 & y_{n-1} & \cdots & d^{n-3} y_{n-1}
\end{array}\right|\right. \\
& \left.\left|\begin{array}{ccccc}
x_{i} & \cdots & d^{j-1} x_{1} & \cdots & d^{n-1} x_{1} \\
\vdots & & \vdots & & \vdots \\
x_{n-1} & \cdots & d^{j-1} x_{n-1} & \cdots & d^{n-1} x_{n-1}
\end{array}\right|\right)
\end{aligned}
$$




$$
\begin{aligned}
& =-\sum_{j=1}^{n}(-1)^{j-1}\left|\begin{array}{cccc}
d^{j-1} y_{1} & y_{1} & \cdots & d^{n-3} y_{1} \\
\vdots & \vdots & & \vdots \\
d^{j-1} y_{i} & y_{i} & \cdots & d^{n-3} y_{i} \\
\vdots & \vdots & & \vdots \\
d^{j-1} y_{n-1} & y_{n-1} & \cdots & d^{n-3} y_{n-1}
\end{array}\right| \\
& \left.\left|\begin{array}{ccccc}
x_{1} & \cdots & d^{j-1} x_{1} & \cdots & d^{n-1} x_{1} \\
\vdots & & \vdots & & \vdots \\
x_{n-1} & \cdots & d^{j-1} x_{n-1} & \cdots & d^{n-1} x_{n-1}
\end{array}\right|\right) \\
& =\left|\begin{array}{cccc}
y_{1} & \cdots & d^{n-3} y_{1} & d^{n-1} y_{1} \\
\vdots & & \vdots & \vdots \\
y_{i} & \cdots & d^{n-3} y_{i} & d^{n-1} y_{i} \\
\vdots & & \vdots & \vdots \\
y_{n-1} & \cdots & d^{n-3} y_{n-1} & d^{n-1} y_{n-1}
\end{array}\right|\left|\begin{array}{ccc}
x_{1} & \cdots & d^{n-2} x_{1} \\
\vdots & & \vdots \\
x_{n-1} & \cdots & d^{n-2} x_{n-1}
\end{array}\right| \\
& -\left|\begin{array}{ccc}
y_{1} & \cdots & d^{n-2} y_{1} \\
\vdots & & \vdots \\
y_{n-1} & \cdots & d^{n-2} y_{n-1}
\end{array}\right|\left|\begin{array}{cccc}
x_{1} & \cdots & d^{n-3} x_{1} & d^{n-1} x_{x} \\
\vdots & & \vdots & \vdots \\
x_{i} & \cdots & d^{n-3} x_{i} & d^{n-1} x_{i} \\
\vdots & & \vdots & \vdots \\
x_{n-1} & \cdots & d^{n-3} x_{n-1} & d^{n-1} x_{n-1}
\end{array}\right| \\
& =\left|\begin{array}{ccc}
x_{1} & \cdots & d^{n-2} x_{1} \\
\vdots & & \vdots \\
x_{n-1} & \cdots & d^{n-2} x_{n-1}
\end{array}\right| d\left|\begin{array}{ccc}
y_{1} & \cdots & d^{n-2} y_{1} \\
\vdots & & \vdots \\
y_{n-1} & \cdots & d^{n-2} y_{n-1}
\end{array}\right| \\
& -\left|\begin{array}{ccc}
y_{1} & \cdots & d^{n-2} y_{1} \\
\vdots & & \vdots \\
y_{n-1} & \cdots & d^{n-2} y_{n-1}
\end{array}\right| d\left|\begin{array}{ccc}
x_{1} & \cdots & d^{n-2} x_{1} \\
\vdots & & \vdots \\
x_{n-1} & \cdots & d^{n-2} x_{n-1}
\end{array}\right| \\
& =\left(\Phi_{\mathcal{L}}^{(n-1)}\left(x_{1} \wedge \cdots \wedge x_{n-1}\right)\right) d\left(\Phi_{\mathcal{L}}^{(n-1)}\left(y_{1} \wedge \cdots \wedge y_{n-1}\right)\right) \\
& -\left(\Phi_{\mathcal{L}}^{(n-1)}\left(y_{1} \wedge \cdots \wedge y_{n-1}\right)\right) d\left(\Phi_{\perp}^{(n-1)}\left(x_{1} \wedge \cdots \wedge x_{n-1}\right)\right) \\
& =\Phi_{\omega_{C}^{\otimes(n-1)(n-2) / 2}(2)}^{\otimes \mathcal{L} \otimes n-1}\left(\Phi_{\mathcal{L}}^{(n-1)}\left(\left[x_{1} \wedge \cdots \wedge x_{n-1}\right]\right)\right. \\
& \left.\wedge \Phi_{\mathcal{L}}^{(n-1)}\left(\left[y_{1} \wedge \cdots \wedge y_{n-1}\right]\right)\right) \text {. }
\end{aligned}
$$

We can calculate the second term in the first equation in the same way, and we are done.

Q.E.D.

LEMMA 2. If $V$ is an $(n+1)$-dimensional vector space, then we have an isomorphism

$$
g: \wedge^{2}\left(\wedge^{n-1} V\right) \oplus \wedge^{n-3} V \longrightarrow \wedge^{n} V \otimes \wedge^{n-2} V
$$


The isomorphism $g$ is given by

$$
\begin{aligned}
g\left(x_{1} \wedge\right. & \left.\cdots \wedge x_{n-1} \wedge y_{1} \wedge \cdots \wedge y_{n-1}, \sigma\right) \\
& =\left[x_{1} \wedge \cdots \wedge x_{n-1}\right] \wedge\left[y_{1} \wedge \cdots \wedge y_{n-1}\right]+\partial \wedge \sigma,
\end{aligned}
$$

where $\partial=\sum_{i=1}^{n}(-1)^{n-i} e_{0} \wedge \cdots \wedge \hat{e}_{i} \wedge \cdots \wedge e_{n} \otimes e_{i}$ and $e_{0}, \cdots, e_{n}$ make a basis of $V$.

PROOF. If $e_{0}, \cdots, e_{n} \in V$ and $0 \leqq i_{0} \leqq n, \cdots, 0 \leqq i_{n} \leqq n$, then

$$
\begin{aligned}
& {\left[e_{i_{0}} \wedge \cdots \wedge e_{i_{n-2}}\right] \wedge\left[e_{i_{n-1}} \wedge e_{i_{0}} \wedge \cdots \wedge e_{i_{n-3}}\right]} \\
& =(-1)^{n} \frac{1}{2}\left\{e_{i_{0}} \wedge \cdots \wedge e_{i_{n-2}} \wedge e_{i_{n-1}}\right) \otimes\left(e_{i_{0}} \wedge \cdots \wedge e_{i_{n-3}}\right. \\
& \quad-\left(e_{i_{n-1}} \wedge e_{i_{0}} \wedge \cdots \wedge e_{i_{n-3}} \wedge(-1)^{n-3} e_{i_{n-2}}\right) \otimes\left(e_{i_{0}} \wedge \cdots \wedge e_{i_{n-3}}\right)\{ \\
& =(-1)^{n}\left(e_{i_{0}} \wedge \cdots \wedge e_{i_{n-1}}\right) \otimes\left(e_{i_{0}} \wedge \cdots \wedge e_{i_{n-3}}\right),
\end{aligned}
$$

and

$$
\begin{aligned}
{\left[e_{i} \wedge\right.} & \left.\cdots \wedge e_{i_{n-2}}\right] \wedge\left[e_{i_{n-1}} \wedge e_{i_{n}} \wedge e_{i_{0}} \wedge \cdots \wedge e_{i_{n-4}}\right] \\
= & (-1)^{n} \frac{1}{2}\left(e_{i_{0}} \wedge \cdots \wedge e_{i_{n-1}}\right) \otimes\left(e_{i_{n}} \wedge e_{i_{0}} \wedge \cdots \wedge e_{i_{n-4}}\right) \\
& -\left(e_{i_{0}} \wedge \cdots \wedge e_{i_{n-2}} \wedge e_{i_{n}}\right) \otimes\left(e_{i_{n-1}} \wedge e_{i_{0}} \wedge \cdots \wedge e_{i_{n-4}}\right) \\
& -\left(e_{i_{n-1}} \wedge e_{i_{n}} \wedge e_{i_{0}} \wedge \cdots \wedge e_{i_{n-4}} \wedge(-1)^{n-} e_{\varepsilon_{i_{n-3}}}\right) \otimes\left(e_{i_{0}} \wedge \cdots \wedge e_{i_{n-4}} \wedge e_{i_{n-3}}\right) \\
& -\left(e_{i_{n-1}} \wedge e_{i_{n}} \wedge e_{i_{0}} \wedge \cdots \wedge e_{i_{n-4}} \wedge(-1)^{n-2} e_{i_{n-2}}\right) \otimes\left(e_{i_{0}} \wedge \cdots \wedge e_{i_{n-4}} \wedge e_{i_{n-3}}\right) \\
= & (-1)^{n} \frac{1}{2}\left(e_{i_{0}} \wedge \cdots \wedge e_{i_{n-1}} \otimes e_{i_{n}}-e_{i_{0}} \wedge \cdots \wedge e_{i_{n-2}} \wedge e_{i_{n}} \otimes e_{i_{n-1}}\right. \\
& -e_{i_{0}} \wedge \cdots \wedge e_{i_{n-3}} \wedge e_{i_{n-1}} \wedge e_{i_{n}} \otimes e_{i_{n-2}} \\
& \left.+e_{i_{0}} \wedge \cdots \wedge e_{i_{n-4}} \wedge e_{i_{n-2}} \wedge e_{i_{n-1}} \wedge e_{i_{n}} \otimes e_{i_{n-3}}\right) \wedge e_{i_{0}} \wedge \cdots \wedge e_{i_{n-4}} \\
= & (-1)^{n} \frac{1}{2}\left(e_{i_{n}}^{*} \otimes e_{i_{n}}+e_{i_{n-1}}^{*} \otimes e_{i_{n-1}}-e_{i_{n-2}}^{*} \otimes e_{i_{n-2}}-e_{i_{n-3}}^{*} \otimes e_{i_{n-3}}\right) e_{i_{0}} \wedge \cdots \wedge e_{i_{n-4}}
\end{aligned}
$$

where $e_{0}^{*}, \cdots, e_{n}^{*} \in \wedge^{n} V \cong V^{*}$ is the dual basis of $e_{0}, \cdots, e_{n}$. Therefore

$$
\begin{aligned}
& {\left[e_{i_{0}} \wedge \cdots \wedge e_{i_{n-2}}\right] \wedge\left[e_{i_{n-1}} \wedge e_{i_{n}} \wedge e_{i_{0}} \wedge \cdots \wedge e_{i_{n-4}}\right]} \\
& \quad=(-1)^{n} \frac{1}{2}\left(e_{i_{n}}^{*} \otimes e_{i_{n}}+e_{i_{n-1}}^{*} \otimes e_{i_{n-1}}-e_{i_{n}{ }_{2}}^{*} \otimes e_{i_{n-2}}-e_{i_{n 3}}^{*} \otimes \partial_{i_{n-3}}\right) \wedge e_{i_{0}} \wedge \cdots \wedge e_{i_{n-4}} \\
& \quad=(-1)^{n}\left(e_{i_{n}}^{*} \otimes e_{i_{n}}+e_{i_{n-1}}^{*} \otimes e_{i_{n-1}}-\frac{1}{2} \partial\right) \wedge e_{i_{0}} \wedge \cdots \wedge e_{i_{n-4}} .
\end{aligned}
$$

Therefore a basis of $\wedge^{n} V \otimes \wedge^{n-2} V$ is contained in $\left.g\left(\wedge^{n-1} V\right) \oplus \wedge^{n-3} V\right)$. Hence $g$ is surjective. Moreover 


$$
\operatorname{dim}_{k}\left(\wedge^{2}\left(\wedge^{n-1} V\right) \oplus V\right)=(n+1) \frac{(n+1) n(n-1)}{3 \cdot 2 \cdot 1} \operatorname{dim}_{k}\left(\wedge^{n} V \otimes \wedge^{n-2} V\right)
$$

Hence $g$ is an isomorphism.

Q.E.D.

LEMMA 3. If $\partial=\sum_{i=0}^{n}(-1)^{n-i} e_{0} \wedge \cdots \wedge \hat{e}_{i} \wedge \cdots \wedge e_{n} \in \wedge{ }^{n} V \otimes V$ where $V$ is an $(n+1)$-dimensional vector subspace of $\Gamma(C, \mathcal{L})$ and $e_{0}, \cdots, e_{n}$ make a basis of $V$, then

$$
\Phi_{\perp}^{(n)} \cap i d_{V}(\partial)=0
$$

Proof. By the definition,

$$
\begin{aligned}
& \Phi_{\mathcal{L}}^{(n)} \cap i d_{V}(\partial)=\sum_{i=0}^{n}(-1)^{n-i} \Phi_{\mathcal{L}}^{(n)}\left(e_{0} \wedge \cdots \wedge \hat{e}_{i} \wedge \cdots \wedge e_{n}\right) e_{i}
\end{aligned}
$$

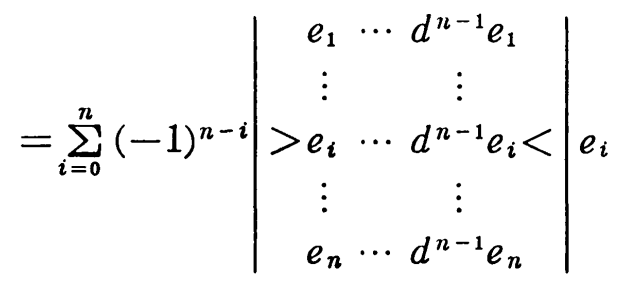

$$
\begin{aligned}
& =(-1)^{n} \sum_{i=0}^{n}\left|\begin{array}{cccc}
0 & e_{1} & \cdots & d^{n-1} e_{1} \\
\vdots & \vdots & & \vdots \\
e_{i} & e_{i} & \cdots & d^{n-1} e_{i} \\
\vdots & \vdots & & \vdots \\
0 & e_{n} & \cdots & d^{n-1} e_{n}
\end{array}\right| \\
& =(-1)^{n}\left|\begin{array}{cccc}
e_{1} & e_{1} & \cdots & d^{n-1} e_{1} \\
\vdots & \vdots & & \vdots \\
e_{i} & e_{i} & \cdots & d^{n-1} e_{i} \\
\vdots & \vdots & & \vdots \\
e_{n} & e_{n} & \cdots & d^{n-1} e_{n}
\end{array}\right| \\
& =0 \text {. }
\end{aligned}
$$

Therefore we have the result.

Q.E.D.

\section{Generalized Castelnuovo's lemma}

In this section, we will give a generalization of Castelnuovo's lemmas in Wahl [18] (p. 86 Theorem 2.6.)

Definition 3. Let $V$ be a vector subspace of $\Gamma(C, \mathcal{L})$ and let $s_{1}, \cdots, s_{N}$ $\in V$ be a basis. For any $p \in C$, if 


$$
\operatorname{rank}\left(\begin{array}{ccc}
s_{1}(p) & \cdots & d^{n} s_{1}(p) \\
\vdots & & \vdots \\
s_{N}(p) & \cdots & d^{n} s_{N}(p)
\end{array}\right)=n+1
$$

then we say that $V$ is $n$-immersive. In particular if $n=1$, then we say that $V$ is immersive.

Definition 4. Let $V$ be a vector subspace of $\Gamma(C, \mathcal{L})$. If $\operatorname{dim}_{k}(V)=n$, then we call that $V$ is an $n$-net.

LEMMA 4. Let $\mathcal{L}$ be an invertible sheaf on a curve $C$ of genus $g$. If $\operatorname{deg}(\mathcal{L})$ $\geqq 2 g+n$, then a general $(n+2)$-dimensional subspace of $\Gamma(C, \mathcal{L})$ is an $n$-immersive $(n+2)-n e t$.

Proof. By the same argument of Hartshorne (see Hartshorne [9] p. 310 Porposition 3.5.), we have

$\operatorname{dim}\left(\bigcup_{p \in C}\left(\right.\right.$ osculating tangent $n$-plaine at $\left.\left.p \in C \subset \boldsymbol{P}\left(\Gamma(C, \mathcal{L})^{*}\right)\right)\right) \leqq n+1$.

This completes the proof.

Q.E.D.

LEMMA 5. Let $\mathcal{L}$ be an invertible sheaf on $C$, and let $V$ be a vector subspace of $\Gamma(C, \mathcal{L})$. If $V$ is an $(n-1)$-immersive, then a sheaf homomorphism

$$
\wedge^{n} V \otimes \mathcal{O}_{C} \longrightarrow \omega_{C}^{\otimes n(n-1) / 2} \otimes \mathcal{L}^{\otimes n}
$$

induced by $\Phi_{V}^{(n)}$ is surjective.

Proof. Let $s_{1}, \cdots, s_{n} \in V$. Then we have

$$
\Phi_{V}^{(n)}\left(s_{1} \wedge \cdots \wedge s_{n}\right)(p)=\left|\begin{array}{ccc}
s_{1}(p) & \cdots & d^{n-1} s_{1}(p) \\
\vdots & & \vdots \\
s_{n}(p) & \cdots & d^{n-1} s_{n}(p)
\end{array}\right|
$$

for every $p \in C$. As $V$ is $(n-1)$-immersive, therefore this completes the proof.

Q.E.D.

The following definition and lemma are famous (see Hizeburch [11]).

DEFinition 5. Let $\mathscr{W}$ and $\mathcal{W}^{\prime}$ be locally free sheaves and let $\mathscr{F}$ be an invertible sheaf. Suppose that a sequence $0 \rightarrow \mathscr{W}^{\prime} \stackrel{\varphi}{\rightarrow} \mathscr{W} \stackrel{\mathscr{\varphi}}{\rightarrow} \mathscr{I} \rightarrow 0$ is exact. The the homomorphism $\wedge^{p} \psi: \wedge^{p} \mathscr{W} \rightarrow \mathscr{F} \otimes \wedge^{p-1} \mathcal{W}^{\prime}$ is defined by 


$$
\wedge^{p} \psi\left(w_{1} \wedge \cdots \wedge w_{p}\right)=\sum_{i=0}^{p}(-1)^{i} \psi\left(w_{i}\right) w_{1} \wedge \cdots \wedge \hat{w}_{i} \wedge \cdots \wedge w_{p}
$$

LEMMA 6. The above $\wedge^{p} \psi$ is surjective and induces an exact sequence

$$
0 \longrightarrow \wedge^{p_{C} W^{\prime}} \stackrel{\wedge^{p} \varphi}{\longrightarrow} \wedge^{p_{\mathcal{W}}} \stackrel{\wedge^{p} \psi}{\longrightarrow} \mathscr{F} \otimes \wedge^{p-1} \mathscr{W} \longrightarrow 0
$$

PROOF. See Hirzeburch [11] p. 55 Theorem 4.1.3. Q.E.D.

Let $V$ be a finite dimensional vector space and $V^{*}$ be a dual vector space of $V$. Let $\partial \in V \otimes V^{*} \cong \operatorname{Hom}_{k}(V, V)$ be an element corresponding to the identity id. We consider the Koszul complex

$$
\mathcal{K}: 0 \longrightarrow \mathcal{O}_{\boldsymbol{P}} \stackrel{\partial}{\longrightarrow} V^{*} \otimes \mathcal{O}_{\boldsymbol{P}}(1) \stackrel{\partial}{\longrightarrow} \wedge^{2} V^{*} \otimes \mathcal{O}_{\boldsymbol{P}}(2) \stackrel{\partial}{\longrightarrow} \cdots
$$

defined by $\partial(f)=f \wedge \partial$ for $f \in \wedge^{i} V^{*} \otimes \mathcal{O}_{\boldsymbol{P}}(i)(i=1,2, \cdots)$ where $\boldsymbol{P}=\boldsymbol{P}\left(V^{*}\right)$.

LEMMA 7. The above Koszul complex $K$ is exact and the image sheaf

$$
\operatorname{im}(\partial)=\operatorname{im}\left(\wedge^{p} V^{*} \otimes \mathcal{O}_{P}(p) \stackrel{\partial}{\longrightarrow} \wedge^{p+1} V^{*} \otimes \mathcal{O}_{P}(p+1)\right)
$$

is isomorphic to $\wedge^{p} T_{\boldsymbol{P}}$, where $T_{\boldsymbol{P}}$ is a tangent sheaf on $\boldsymbol{P}$.

Proof. If $p=1$, this is obvious (for example, see Hartshorne [9] p. 176). If $p$ is arbitary, then this follows directly from Lemma 6.

Q.E.D.

Definition 6 . Let $V$ be an $(n-1)$-immersive $(n+1)$-net. A locally free sheaf $Q_{n}$ is given by

$$
Q_{n}=\operatorname{ker}\left(\wedge^{n} V \otimes \mathcal{O}_{C} \rightarrow \omega_{C}^{\otimes n(n-1) / 2} \otimes \mathcal{L}^{\otimes n}\right)
$$

REMARK 1. As $\Phi_{\mathcal{L}}^{(n)}: \wedge^{n} V \otimes \mathcal{O}_{C} \rightarrow \omega_{C}^{\otimes n(n-1) / 2} \otimes \mathcal{L}^{\otimes n}$ is surjective, $Q_{n}$ is a locally free sheaf of rank $n$.

Let $V$ be an $(n-1)$-immersive $(n+1)$-net. As $V^{*} \cong \wedge^{n} V, \quad \partial \in V^{*} \otimes V$ is given by

$$
\partial=\sum_{i=0}^{n}(-1)^{n-i} e_{0} \wedge \cdots \wedge \hat{e}_{i} \wedge \cdots \wedge e_{n} \otimes e_{i}
$$

Therefore $\partial$ is contained in $\Gamma\left(C, Q_{n}\right)$ by Lemma 3 in $\S 1$. As $V$ is $(n-1)$ immersive, $V$ is base point free, so $V$ defines a morphism $C \rightarrow \boldsymbol{P}\left(V^{*}\right)$. We restrict the above Koszul complex $\mathcal{K}$ to $C \rightarrow \boldsymbol{P}\left(V^{*}\right)$ and we have the following exact sequence : 


$$
\text { (B) }: 0 \longrightarrow \mathcal{O}_{C} \stackrel{\partial}{\longrightarrow} V^{*} \otimes \mathcal{L} \stackrel{\partial}{\longrightarrow} \wedge^{2} V^{*} \otimes \mathcal{L} \stackrel{\partial}{\longrightarrow} \cdots .
$$

As $\partial \in \Gamma\left(C, Q_{n}\right), \partial$ defines the following complex:

$$
(\mathrm{A}): 0 \longrightarrow \mathcal{O}_{C} \stackrel{\partial}{\longrightarrow} Q_{n} \otimes \mathcal{L} \stackrel{\partial}{\longrightarrow} \wedge^{2} Q_{n} \otimes \mathcal{L} \stackrel{\partial}{\longrightarrow} \cdots .
$$

where the map $\partial$ is defined qy $\partial(f)=f \wedge \partial$. Moreover we consider a complex $(C)=(A) \otimes \omega_{C}^{\otimes n(n-1) / 2} \otimes \mathcal{L}^{\otimes n}:$

$$
\begin{aligned}
0 \longrightarrow \omega_{C}^{\otimes n(n-1) / 2} \otimes \mathcal{L}^{\otimes n} & \stackrel{\partial}{\longrightarrow} Q_{n} \otimes \omega_{C}^{\otimes n(n-1) / 2} \otimes \mathcal{L}^{\otimes n+1} \\
& \stackrel{\partial}{\longrightarrow} \wedge^{2} Q_{n} \otimes \omega_{C}^{\otimes n(n-1) / 2} \otimes \mathcal{L}^{\otimes n+2} \ldots
\end{aligned}
$$

By Definition 6, we have the following short exact sequence:

$$
(1): 0 \longrightarrow Q_{n} \otimes \mathcal{L} \stackrel{\varphi}{\longrightarrow} V^{*} \otimes \mathcal{L} \longrightarrow \omega_{C}^{\otimes n(n-1) / 2} \otimes \mathcal{L}^{\otimes n+1} \longrightarrow 0 .
$$

Therefore we have the following short exact sequences by Lemma 6:

$$
\text { (i) : } 0 \rightarrow \wedge^{i} Q_{n} \otimes \mathcal{L}^{\otimes i} \stackrel{\wedge^{i} \varphi}{\longrightarrow} \wedge^{i} V^{*} \otimes \mathcal{L}^{\otimes i} \stackrel{\wedge^{i} \psi}{\longrightarrow} \omega_{C}^{\otimes n(n-1) / 2} \otimes \mathcal{L}^{\otimes n+i} \otimes \wedge^{i-1} Q_{n} \rightarrow 0
$$

where $i=1,2, \cdots$. Hence we have the following diagram:

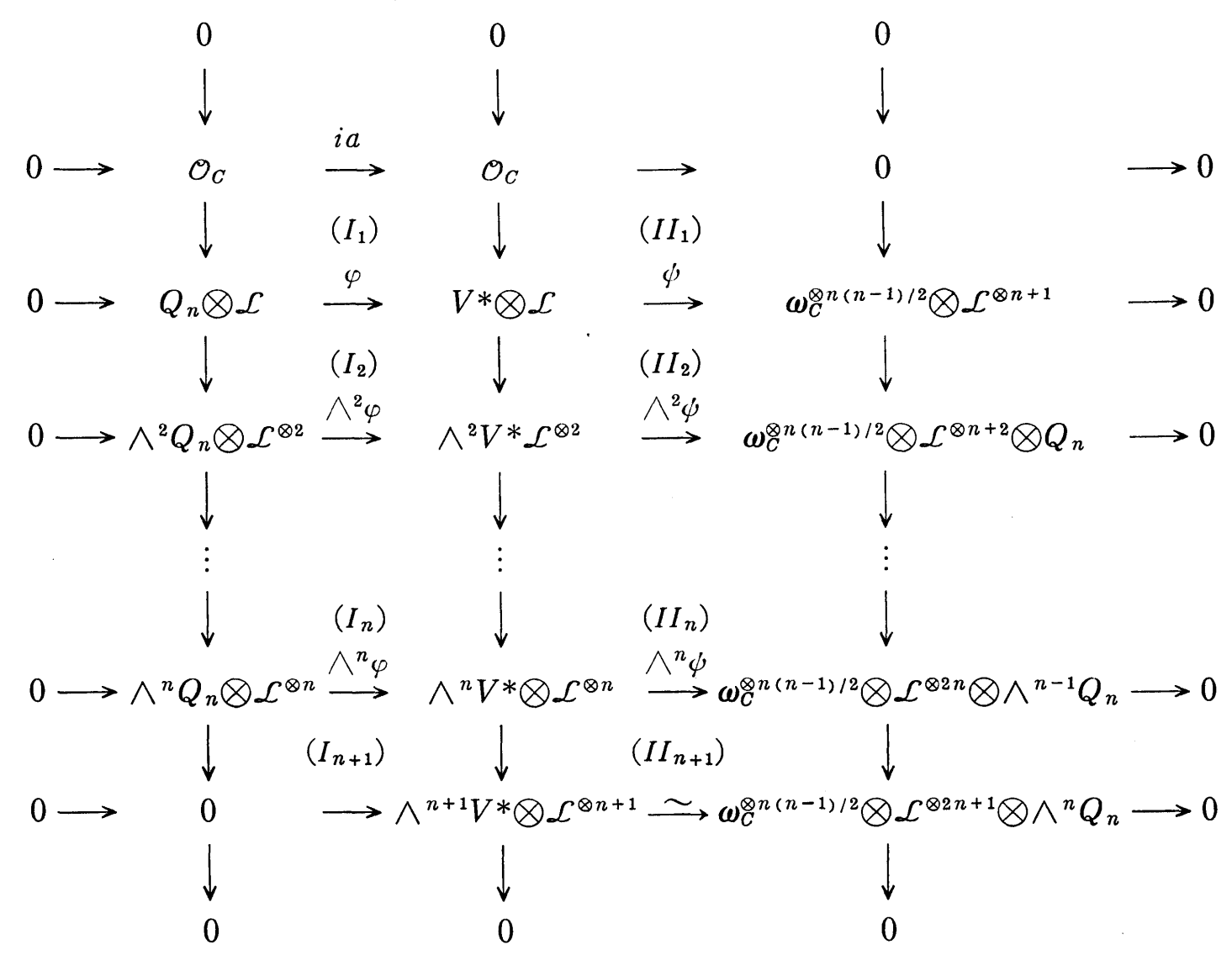


where $\phi=\Phi_{\perp}^{(n)}$.

LEMMA 8. The above diagram is commutative.

Proof. It is easy that $\left(I_{i}\right)$ part is commutative for $i=1,2, \ldots$. Because

$$
)\left(\wedge^{i-1} \varphi\right)\left(x_{i} \wedge \cdots \wedge x_{i-1}\right)\right) \wedge \partial=\left(\wedge^{i} \varphi\right)\left(x_{1} \wedge \cdots \wedge x_{i-1} \wedge \partial\right)
$$

by the definition of (A) and (B). We now show that $\left(I I_{i}\right)$ part is commutative for $i=1, z, \cdots$. This is equivalent to

$$
\left(\left(\wedge^{i-1} \psi\right)\left(x_{i} \wedge \cdots \wedge x_{i-1}\right)\right) \wedge \partial=\left(\wedge^{i} \psi\right)\left(x_{1} \wedge \cdots \wedge x_{i-1} \wedge \partial\right)
$$

for $i=1,2, \cdots$. As

$$
\left(\wedge^{i-1} \psi\right)\left(x_{i} \wedge \cdots \wedge x_{i-1}\right) \wedge \partial=\sum_{j=1}^{i-1}(-1)^{j} \psi\left(x_{j}\right) x_{1} \wedge \cdots \wedge \hat{x}_{j} \wedge \cdots \wedge x_{i=1} \wedge \partial,
$$

and

$$
\begin{aligned}
& \left(\wedge^{i} \psi\right)\left(x_{1} \wedge \cdots \wedge x_{i-1} \wedge \partial\right) \\
& \quad=\sum_{j=1}^{i-1}(-1)^{j} \psi\left(x_{j}\right) x_{1} \wedge \cdots \wedge \hat{x}_{j} \wedge \cdots \wedge x_{i-1} \wedge \partial+(-1)^{i} \psi(\partial) x_{1} \wedge \cdots \vee x_{i-1} \\
& \quad=\sum_{j=1}^{i-1}(-1)^{j} \psi\left(x_{j}\right) x_{1} \wedge \cdots \wedge \hat{x}_{j} \wedge \cdots \wedge x_{i-1} \wedge \partial
\end{aligned}
$$

we have the commutativity of $\left(I I_{i}\right)$ by Lemma 3 .

Q.E.D.

LEMMA 9. The complexes (A) and (B) are exact.

Proof. As the exact sequence (1) splits locally, there is a local section $\iota_{1}$ of $\varphi$. We put $\iota_{i}=\wedge^{i} \iota_{c}(i=0,1, \cdots)$ and put $\varphi_{i}=\wedge^{i} \varphi(i=0,1, \cdots)$. By the definition of $\iota_{1}$, it is clear that $\partial \iota_{0}=\iota_{1} \partial$. Therefore we have $\iota_{m+1} \partial=\partial \iota_{m}$ and $\iota_{m} \varphi_{m}=i d$. Hence the exact sequence of complexes

$$
0 \longrightarrow(\mathrm{A}) \longrightarrow(\mathrm{B}) \longrightarrow(\mathrm{C}) \longrightarrow 0
$$

splits locally. As (B) is exact, therefore (A) and (C) are exact complexes.

Q.E.D.

LEMMA 10. Let $V$ be a subspace of $\Gamma(C, \mathcal{L})$ with $\operatorname{dim}_{k} V \geqq n+1$. If $V$ is $(n+1)$-immersive, then the subspace

is immersive.

$$
\Phi_{\mathcal{L}}^{(n-1)}\left(\wedge^{n-1} V\right) \subset \Gamma\left(C, \omega_{C}^{\otimes n(n-1) / 2} \otimes \mathcal{L}^{\otimes n-1}\right)
$$

Proof. Let $x_{1}, \cdots, x_{n-1} \in V$. Then 


$$
\begin{aligned}
& \Phi_{\omega_{C}^{\otimes n(n-1) / 2} \otimes \mathcal{L}^{n-1}}^{(2)}\left(\Phi_{\mathcal{L}}^{(n-1)}\left(x_{1} \wedge \cdots \wedge x_{n-1}\right) \wedge \Phi_{\perp}^{(n-1)}\left(x_{n} \wedge x_{1} \wedge \cdots \wedge x_{n-2}\right)\right) \\
& =\left|\begin{array}{ccc}
x_{1} & \cdots & d^{n-1} x_{1} \\
\vdots & & \vdots \\
x_{n} & \cdots & d^{n-1} x_{n}
\end{array}\right|\left|\begin{array}{ccc}
x_{1} & \cdots & d^{n-3} x_{1} \\
\vdots & & \vdots \\
x_{n-2} & \cdots & d^{n-3} x_{n-2}
\end{array}\right| .
\end{aligned}
$$

Hence for every $p \in C$ there are some $v, v^{\prime} \in \wedge^{n-1} V$ such that

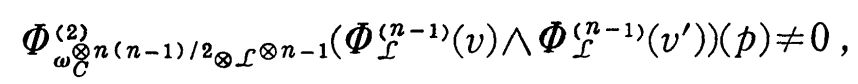

because $V$ is $(n-1)$-immersive. Therefore we have the result.

Q.E.D.

LEMMA 11. Let $\mathcal{E}$ be a locally free sheaf on $C$, let $V$ be a subspace of $\Gamma(C, \mathcal{E})$ and let $K$ be a function field of $C$. If the canonical map $V \otimes_{k} K \rightarrow$ $\tilde{\mathcal{E}} \otimes{ }_{\sigma_{C}} K$ is injective, then $V \otimes_{k} \mathcal{O}_{C}$ is subsheaf of $\mathcal{E}$.

PRFOO. This is obvious.

Q.E.D.

LEMMA 12. Let $V$ be a subspace of $\Gamma(C, \mathcal{L})$ with $\operatorname{dim}_{k} V=n+1$. If $W_{0}$ is a general 3-dimensional linear subspace of $\wedge^{n-1} V$, then a composition of two canonical maps

$$
\wedge^{2} W_{0} \otimes \mathcal{O}_{C} \longrightarrow \wedge^{n} V \otimes \wedge^{n-2} V \otimes \mathcal{O}_{C} \longrightarrow T_{P^{n}{ }_{1}} \otimes \omega_{C}^{\otimes(n-2)(n-3) / 2} \otimes \mathcal{L}^{\otimes n-3}
$$

is injective.

Proof. This condition is an open condition. Therefore we construct an example of $W_{0}$ which satisfies the property of this lemma. Let $x_{1}, \cdots, x_{n+1}$ be a basis of $V$ and let $W_{0}=\left[x_{1} \wedge \cdots \wedge x_{n-1}, x_{n} \wedge x_{1} \wedge \cdots<x_{n-2}, x_{n+1} \wedge x_{1} \wedge \cdots \wedge\right.$ $\left.x_{n-2}\right]$. By Lemma 2, a basis of $\Phi_{\mathcal{L}}^{(n)} \cap \Phi_{\perp}^{(n-2)}\left(\wedge^{2} W_{0}\right)$ is $\left(x_{1} \wedge \cdots \wedge x_{n}\right) \otimes\left(x_{1} \wedge \cdots\right.$ $\left.\wedge x_{n-2}\right), \quad\left(x_{1} \wedge \cdots \wedge x_{n-1} \wedge x_{n+1}\right) \otimes\left(x_{1} \wedge \cdots \wedge x_{n-2}\right), \quad\left(x_{1} \wedge \cdots \wedge x_{n-2} \wedge x_{n} \wedge x_{n+1}\right) \otimes$ $\left(x_{1} \wedge \cdots \wedge x_{n-2}\right)$. Therefore we can construct an example of $W_{0}$. Q.E.D.

LEMMA 13. Let $V$ be (n-1)-immersive and $\operatorname{dim}_{k}(V) \geqq n+1$. If $W_{0}$ is $a$ general 3-dimensional linear subspace of $\wedge^{n-1} V$, then $\Phi_{\mathcal{L}}^{(n-1)}\left(W_{0}\right)$ is an immersive net.

Proof. This condition is also an open condition. Therefore this lemma $\begin{array}{ll}\text { follows from Lemma 10. } & \text { Q.E.D. }\end{array}$

LEMMA 14. If $V \subset \wedge^{n-1} V$ is an $(n-1)$-immersive $(n+1)$-net, then there is an $n$-dimensional subspace $W \subset \wedge^{2}\left(\wedge^{n-1} V\right)$ such that

(1) there is $W_{0} \subset \wedge^{n-1} V$ such that $\operatorname{dim}_{k} W_{0}=3, \wedge^{2} W_{0} \subset W$ and $\Phi_{\mathcal{L}}^{(2)}\left(W_{0}\right)$ is im- 
mersive net,

(2) $\wedge^{2} W_{0} \otimes \mathcal{O}_{C} \rightarrow \wedge{ }^{n} V \otimes \wedge^{n-2} V \otimes \mathcal{O}_{C} \leftarrow T_{P^{n}{ }_{1}} \otimes \omega_{C}^{\otimes(n-2)(n-3) / 2} \otimes \mathcal{L}^{\otimes n-3}$ is injective.

Proof. By Lemma 12 and Lemma 13, there is a 3-dimensional subspace $W_{0} \subset \wedge^{n-1} V$ such that

(1) there is $W_{0} \subset \wedge^{n-1} V$ such that $\operatorname{dim}_{k} W_{0}=3$ and $\Phi_{\mathcal{L}}^{(2)}\left(W_{0}\right)$ is immersive net,

(2) $\wedge{ }^{2} W_{0} \otimes \mathcal{O}_{C} \rightarrow \wedge{ }^{n} V \otimes \wedge{ }^{n-2} V \otimes \mathcal{O}_{C} \rightarrow T_{P n_{1} C} \otimes \omega_{C(n-2)(n-3) / 2}^{\otimes} \otimes \mathcal{L}^{\otimes n-3}$ is injective. As $0 \rightarrow \mathcal{O}_{C} \rightarrow \wedge^{n} V \otimes \mathcal{L} \rightarrow T_{P n_{1} C} \rightarrow 0$ is some $\bar{W} \subset \wedge^{n} V \otimes \wedge{ }^{n-2} V$ such that $\left(\wedge^{2} W_{0} \oplus \bar{W}\right)$ $\otimes K=T_{\boldsymbol{P}_{n_{1} C}} \otimes_{\mathcal{O}_{C}} \otimes K$. We put $W=\wedge^{2} W_{0} \oplus \bar{W}$. By Lemma 11, we get the result.

Q.E.D.

Definition 7. Let $V \subset \Gamma(C, \mathcal{L})$ be an $(n-1)$-immersive $(n+1)$-net. By the following exact commutative diagrams, we define a locally free sheaf $\mathcal{E}_{n}$ :

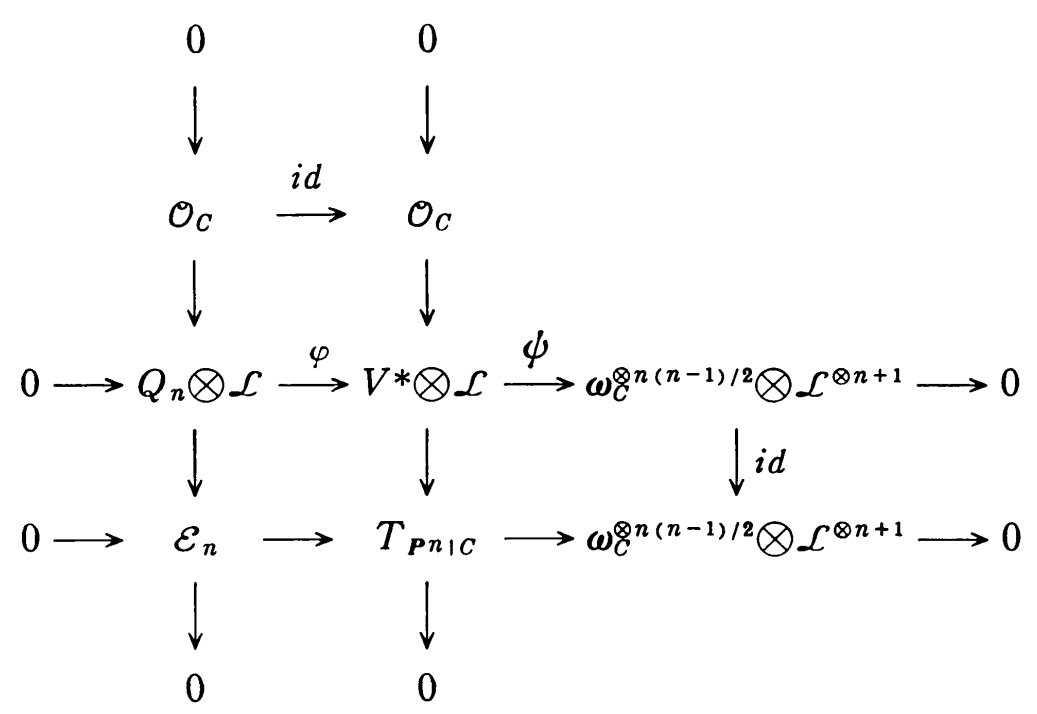

Let $W_{0} \subset \wedge^{n-1} V$ and $W \subset \wedge^{2}\left(\wedge^{n-1} V\right)$ be vector subspaces in Lemma 14 . By the following exact sequences, we define locally free shəaves $Q_{W_{0}}$ and $Q_{0}$ :

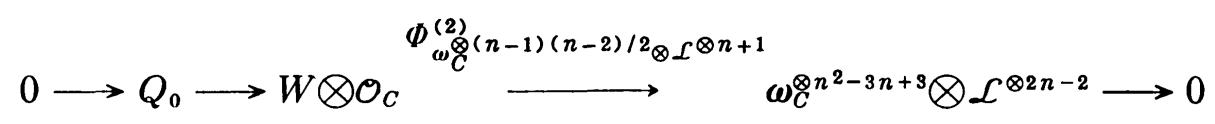

and

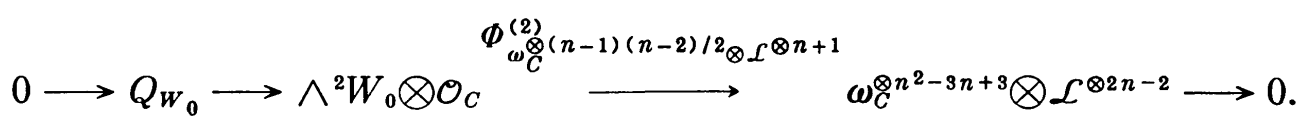

PROPOSITION 1. We have the following exact sequences:
$(\mathrm{A}): 0 \longrightarrow \mathrm{O}_{C} \longrightarrow Q_{n} \otimes \mathcal{L} \longrightarrow \mathcal{E}_{n} 0$
(B) : $0 \longrightarrow Q_{0} \longrightarrow \mathcal{E}_{n} \otimes \omega_{C}^{\otimes(n-2)(n-3) / 2} \otimes \mathcal{L}^{\otimes n-3} \longrightarrow($ torsion sheaf $) \longrightarrow 0$ 


$$
\begin{aligned}
& \qquad \begin{array}{l}
(\mathrm{C}): 0 \longrightarrow Q_{W_{0}} \longrightarrow Q_{0} \longrightarrow \bar{W} \otimes \mathcal{O}_{C} \longrightarrow 0 \\
(\mathrm{D}): 0 \longrightarrow\left(\omega_{C}^{\otimes(n-1)(n-2) / 2} \otimes \mathcal{L}^{\otimes n-1}\right)^{\otimes-1} \longrightarrow Q_{W_{0}} \\
\text { where } \bar{W}=W /\left(\wedge^{2} W_{0}\right) .
\end{array} \quad \longrightarrow\left(\omega_{C}^{\otimes 1+((n-1)(n-2)) / 2} \otimes \mathcal{L}^{\otimes u-1}\right)^{\otimes-1} \longrightarrow 0
\end{aligned}
$$

Proof. The sequence (A) is given by the definition of $\mathcal{E}_{n}$. Now we consider the sequence (B). By Lemma 14, we have the following commutative exact diagram :

$$
\begin{aligned}
& 0 \longrightarrow \mathcal{E}_{n} \otimes \omega_{C}^{\otimes(n-2)(n-3) / 2} \otimes \mathcal{L}^{\otimes n-3} \longrightarrow \\
& 0 \longrightarrow \quad Q_{0} \quad \longrightarrow \\
& T_{\boldsymbol{P}{ }^{\prime} \mid C} \otimes \omega_{G}^{\otimes(n-2)(n-3) / 2} \otimes \mathcal{L}^{\otimes n-3} \longrightarrow \omega_{C}^{\otimes n^{2}-3 n+3} \otimes \mathcal{L}^{\otimes 2 n-2} \longrightarrow 0 \\
& \uparrow \text { inclusion } \uparrow \text { id } \\
& W \otimes \mathcal{O}_{C} \longrightarrow \quad \omega_{C}^{\otimes n^{2}-3 n+3} \otimes \mathcal{L}^{\otimes 2 n-2} \longrightarrow 0 \\
& \Phi_{\omega_{C}^{\otimes}(n-1)(n-2) / 2}^{(2)} \otimes \mathcal{L}^{\otimes n+1}
\end{aligned}
$$

Therefore there is an injective morphism:

$$
Q_{0} \longrightarrow \mathcal{E}_{n} \otimes \omega_{C}^{\otimes(n-2)(n-3) / 2} \otimes \mathcal{L}_{\otimes}^{n-3} \cdot
$$

As $Q_{0}$ and $\mathcal{E}_{n} \otimes \omega_{C}^{\otimes(n-2)(n-3) / 2} \otimes \mathcal{L}^{\otimes n-3}$ are both locally free invertible sheaves of rank $n-1$, hence we have (B). By the exact sequence

$$
0 \longrightarrow Q_{0} \longrightarrow W \otimes \mathcal{O}_{C} \longrightarrow \omega_{C}^{\otimes n^{2}-3 n+3} \otimes \mathcal{L}^{\otimes 2 n-2} \longrightarrow 0,
$$

the following exact commutative diagram is obtained:

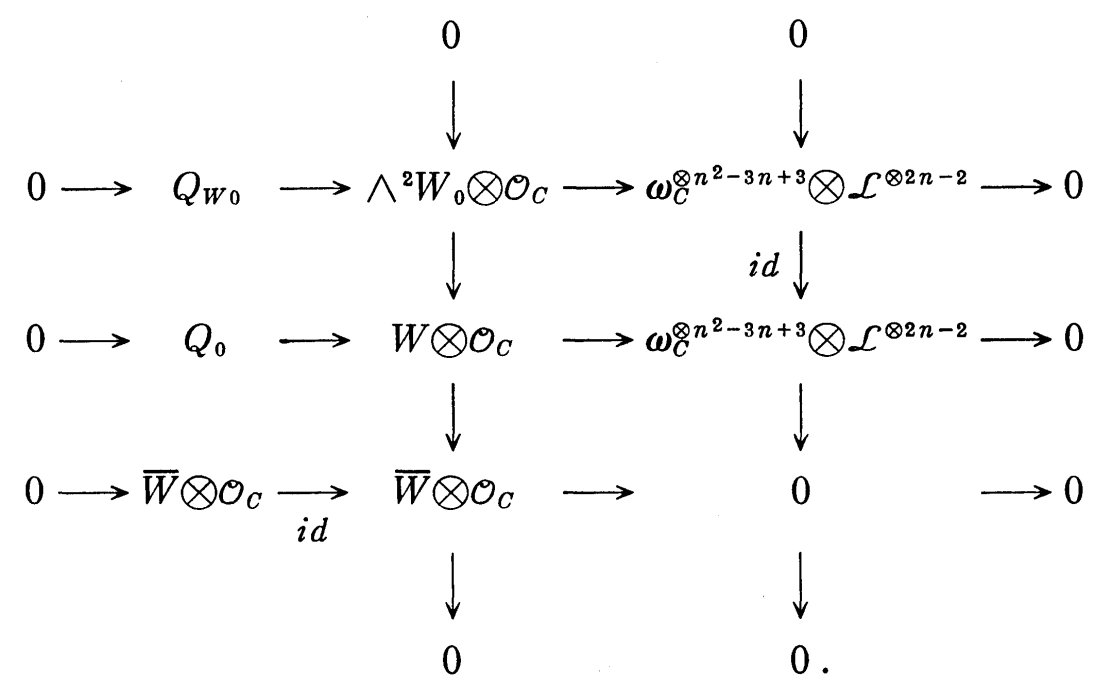

Therefore we have the exact sequence (C) by the snake lemma. As $W_{0}$ is an immersive net, the exact sequence (D) is obtained by the definition of $Q_{2}$ (see 
Wahl [18] p. 85 Lemma 2.4.).

Q.E.D.

By these lemmas and proposition, we have the following theorem which is a generalization of Castelnuovo's lemma in [18].

THEOREM 1. If $V \subset \Gamma(C, \mathcal{L})$ is an $(n-1)$-immersive $(n+1)$-net and if $\mathcal{F}$ is a coherent $\mathcal{O}_{C}$-module on $C$ such that

$$
\begin{aligned}
& H^{1}\left(C, \mathcal{L}^{\otimes-2} \otimes \mathscr{I}\right)=0, \\
& H^{1}\left(C,\left(\omega_{\mathcal{C}}^{\otimes(n-2)(n-3) / 2} \otimes \mathcal{L}^{\otimes n-1}\right)^{\otimes-1} \otimes \mathscr{I}\right)=0, \\
& H^{1}\left(C,\left(\omega_{C}^{\otimes^{n 2-4 n+4}} \otimes \mathcal{L}^{\otimes 2 n-2}\right)^{\otimes-1} \otimes \mathscr{I}\right)=0, \\
& H^{1}\left(C,\left(\omega_{C}^{\otimes n^{2}-4 n+5} \otimes \mathcal{L}^{\otimes 2 n-2}\right)^{\otimes-1} \otimes \mathscr{I}\right)=0,
\end{aligned}
$$

then a canonical map

$$
\wedge^{n} V \otimes \Gamma\left(C, \mathcal{L}^{\otimes-1} \otimes \mathscr{I}\right) \longrightarrow \Gamma\left(C, \omega_{C}^{\otimes n(n-1) / 2} \otimes \mathcal{L}^{\otimes n-1} \otimes \mathscr{I}\right)
$$

which is induced by an $n$-Wahl map $\Phi_{\mathcal{L}}^{(n)}$ is surjective.

PROOF. We put $(A)_{1}=(A) \otimes \mathcal{L}^{\otimes-2} \otimes \mathscr{F}, \quad(B)_{1}=(B) \otimes\left(\omega_{C}^{\otimes(n-2)(n-3) / 2} \otimes \mathcal{L}^{\otimes n-1}\right)^{\otimes-1}$ $\otimes \mathscr{F},(C)_{1}=(C) \otimes\left(\omega_{C}^{\otimes(n-2)(n-3) / 2} \otimes \mathcal{L}^{\otimes n-1}\right)^{\otimes-1} \otimes \mathscr{F},(D)_{1}=(D) \otimes\left(\omega_{C}^{\otimes(n-2)(n-3) / 2} \otimes \mathcal{L}^{\otimes n-1}\right)^{\otimes-1}$ $\otimes \mathscr{F}$. By $(B)_{1}$, we have the following two exact sequences:

$$
\begin{gathered}
0 \longrightarrow(\text { kernel }) \longrightarrow Q_{0} \otimes\left(\omega_{C}^{\otimes(n-2)(n-3) / 2} \otimes \mathcal{L}^{\otimes n-3}\right)^{\otimes-1} \mathscr{T} \longrightarrow(\text { image }) \longrightarrow 0 \\
0 \longrightarrow(\text { image }) \longrightarrow \mathcal{E}_{n} \otimes \mathcal{L}^{\otimes-2} \otimes \mathscr{I} \longrightarrow(\text { torsion sheaf }) \longrightarrow 0 .
\end{gathered}
$$

By the exact sequence $(D)_{1}$ and an assumption, we have that

$$
H^{1}\left(C, Q_{W_{0}} \otimes\left(\omega_{C}^{\otimes(n-2)(n-3) / 2} \otimes \mathcal{L}^{\otimes n-1} \otimes \mathscr{I}\right)=0 .\right.
$$

Therefore we have

$$
H^{1}\left(C, Q_{0} \otimes\left(\omega_{C}^{\otimes(n-2)(n-3)} \otimes \mathcal{L}^{\otimes n-1}\right)^{\otimes-1} \otimes \mathscr{F}\right)=0
$$

by the exact sequence $(C)_{1}$. Hence

$$
H^{1}\left(C, \mathcal{E}_{n} \otimes \mathcal{L}^{\otimes-2} \otimes \mathscr{F}\right)=0
$$

by the exact sequence $(B)_{1}$ because $C$ is one-dimensional. And

$$
H^{1}\left(C, Q_{n} \otimes \mathcal{L}^{\otimes-1} \otimes \mathscr{I}\right)=0
$$

by the sequence $(A)_{1}$. Therefore we have the result.

Q.E.D. 


\section{Application}

In this section, we consider the surjectivity of $\Phi_{\perp}^{(n)}$. Now we prepare several definitions and lemmas.

Definition 8. Let $\mathcal{L}_{1}, \cdots, \mathcal{L}_{n}$ be invertible sheaves on $C$. We define a vector subspace $R\left(\mathcal{L}_{1}, \cdots, \mathcal{L}_{n}\right) \subset \Gamma\left(C, \mathcal{L}_{1}\right) \otimes \cdots \otimes \Gamma\left(C, \mathcal{L}_{n}\right)$ by

$$
\left\{\begin{array}{r}
\sum_{j} x_{j}^{(\sigma(1))}\left(d^{i_{1}} x_{j}^{(\sigma(2))}\right) \cdots\left(d^{i_{n-1}} x_{j}^{(\sigma(n))}\right)=0 \\
\left.\sum_{j} x_{j}^{(1)} \otimes \cdots \otimes x\right\}^{(n)}: 0 \leqq i_{1} \leqq 1, \cdots, 0 \leqq i_{n-1} \leqq n-1 \\
\text { and } \sum_{s=1}^{n-1} i_{s}<\frac{n(n-1)}{2}, \text { for any } \sigma \in S_{n}
\end{array}\right\} .
$$

LEMMA 15. The above space $\mathscr{R}\left(\mathcal{L}_{1}, \cdots, \mathcal{L}_{n}\right)$ is well-defined.

Proof. Let $f_{i}$ be a transition function of an invertible sheaf $\mathcal{L}_{i}$ and let $\tilde{x}_{j}^{(i)}=x_{j}^{(i)} f_{i}$ where $x_{j}^{i}$ and $\tilde{x}_{j}^{i}$ are local sections of $\mathcal{L}_{i}(i=1, \cdots)$. We assume that $\sum_{j} x_{j}^{(1)} \otimes \cdots \otimes x_{j}^{(n)}$ satisfies

$$
\sum_{j} x_{j}^{(\sigma(1))}\left(d^{i_{1}} x_{j}^{(\sigma(2))}\right) \cdots\left(d^{i_{n-1}} x_{j}^{(\sigma(n))}\right)=0
$$

for $0 \leqq i_{1} \leqq 1, \cdots, 0 \leqq i_{n-1} \leqq n-1, \sum_{s=1}^{n-1} i_{s}<\frac{n(n-1)}{2}$ and $\sigma \in S_{n}$. As

$$
d^{i}(f x)=\sum_{i=0}^{i}\left(\begin{array}{l}
i \\
s
\end{array}\right)\left(d^{s} x\right)\left(d^{n-s} f\right),
$$

we have that

$$
\begin{aligned}
\sum_{j} \tilde{x}_{j}^{(\sigma(1))}\left(d^{i_{1}} \tilde{x}_{j}^{(\sigma(2))}\right) \cdots\left(d^{i_{n-1}} \tilde{x}_{j}^{(\sigma(n))}\right. \\
=\sum_{s_{1}=0}^{i_{1}} \cdots \sum_{s_{n-1}=0}^{i_{n-1}}\left(\sum_{j} x_{j}^{(\sigma(1))}\left(d^{i_{1}} x j^{\sigma(2))}\right) \cdots\left(d^{i_{n-1}} x_{j}^{(\sigma(n))}\right)\right) \\
\quad \cdot\left(\begin{array}{c}
i_{1} \\
s_{1}
\end{array}\right) \cdots\left(\begin{array}{c}
i_{n-1} \\
s_{n-1}
\end{array}\right) f_{\sigma(1)}\left(d^{i_{1}-s_{1}} f_{\sigma(\sigma)}\right) \cdots\left(d^{i_{n-1}-s_{n-1}} f_{\sigma(n)}\right)
\end{aligned}
$$

Therefore we have the result.

Q.E.D.

REMARK 2. If $n=2$, then $\mathscr{R}\left(\mathcal{L}_{1}, \mathcal{L}_{2}\right)$ in Definition 8 is the kernel of a cup product map

$$
\Gamma\left(C, \mathcal{L}_{1}\right) \otimes \Gamma\left(C, \mathcal{L}_{2}\right) \longrightarrow \Gamma\left(C, \mathcal{L}_{1} \otimes \mathcal{L}_{2}\right)
$$

because

$$
\sum_{j} x_{j}^{(1)} x_{j}^{(2)}=0
$$


in the only relation in Definition 8.

Definition 9. Let $\tau=\sum x_{j}^{(1)} \otimes \cdots \otimes x_{j}^{(n)} \in \mathcal{R}\left(\mathcal{L}_{1}, \cdots, \mathcal{L}_{n}\right)$. We define the $n$-Wahl map

$$
\Phi_{\mathcal{L}_{1}, \cdots, \mathcal{L}_{n}}: \mathscr{R}\left(\mathcal{L}_{1}, \cdots, \mathcal{L}_{n}\right) \longrightarrow \Gamma\left(C, \omega_{C}^{\otimes n(n-1) / 2} \otimes \mathcal{L}_{1} \otimes \cdots \otimes \mathcal{L}_{n}\right)
$$

by

$$
\begin{aligned}
\Phi_{\mathcal{L}_{1}, \cdots, \mathcal{L}_{n}}(\tau) & =\Phi_{\mathcal{L}_{1}, \cdots, \mathcal{L}_{n}}\left(\sum_{j} x_{j}^{(1)} \otimes \cdots \otimes x_{j}^{(n)}\right) \\
& =\frac{1}{n !} \sum_{j}\left(\sum_{\sigma \in \Phi_{n}} \operatorname{sgn}(\sigma) x_{j}^{(\sigma(1))}\left(d x_{j}^{(\sigma(2))}\right) \cdots\left(d^{n-1} x_{j}^{(\sigma(n))}\right)\right) .
\end{aligned}
$$

LEMMA 16. The above $\Phi_{\mathcal{L}_{1}, \ldots, \mathcal{L}_{n}}$ is well-defined.

Proof. Let $f_{i}$ be a transition function of an invertible sheaf $\mathcal{L}_{i}$ and let $\tilde{x}_{j}^{(i)}=x_{j}^{(i)} f_{i}(i=1, \cdots)$. Then

$$
\begin{aligned}
& \sum_{j}\left(\sum_{\sigma \in \Theta_{n}} \operatorname{sgn}(\sigma) \tilde{x}_{j}^{(\sigma(1))}\left(d \tilde{x}_{j}^{(\sigma(2))}\right) \cdots\left(d^{n-1} \tilde{x}_{j}^{(\sigma(n))}\right)\right) \\
& =\sum_{j}\left(\sum_{\sigma \in \subseteq_{n}} \operatorname{sgn}(\sigma) x_{j}^{(\sigma(1))}\left(d x_{j}^{(\sigma(2))}\right) \cdots\left(d^{n-1} x_{j}^{(\sigma(n))}\right)\right)\left(f_{1} \cdots f_{n}\right) \\
& +\sum_{j}\left(\sum _ { \sigma \in \Theta _ { n } } \left(_{u_{2}+v_{2}=1, v_{2} \neq 1} \cdots \sum_{u_{n}+v_{n}=n-1, v_{n} \neq n-1} \operatorname{sgn}(\sigma)\right.\right. \\
& \left.\left.f_{\sigma(1)} x_{j}^{(\sigma(1))}\left(\begin{array}{c}
1 \\
u_{2}
\end{array}\right)\left(d^{u_{2}} f_{\sigma(2)}\right)\left(d^{v_{2}} x_{j}^{(\sigma(2))}\right) \cdots\left(\begin{array}{c}
n-1 \\
u_{n}
\end{array}\right)\left(d^{u_{n}} f_{\sigma(n)}\right)\left(d^{v_{n}} x_{j}^{(\sigma(n))}\right)\right)\right) \\
& =\sum_{j}\left(\sum_{\sigma \in \mathscr{S}_{n}} \operatorname{sgn}(\sigma) x_{j}^{(\sigma(1))}\left(d x_{j}^{(\sigma(2))}\right) \cdots\left(d^{n-1} x_{j}^{(\sigma(n))}\right)\right)\left(f_{1} \cdots f_{n}\right) \text {. }
\end{aligned}
$$

Thus we have the result.

Q.E.D.

LemMA 17. Assume that $x_{1}, \cdots, x_{n} \in \Gamma(C, \mathcal{L})$, then $\mathscr{R}(\mathcal{L}, \cdots, \mathcal{L})$ contains

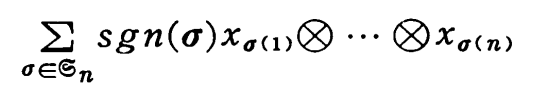

and

$$
\Phi_{\mathcal{L}_{1}, \cdots, \mathcal{L}_{n}}\left(\sum_{\sigma \in \Theta_{n}} \operatorname{sgn}(\sigma) x_{\sigma(1)} \otimes \cdots \otimes x_{\sigma(n)}\right)=\Phi_{\mathcal{L}}^{(n)}\left(x_{1} \wedge \cdots \wedge x_{n}\right) .
$$

PROOF. If $0 \leqq i_{1} \leqq 1, \cdots, 0 \leqq i_{n-1} \leqq n-1$ and $\sum_{s=1}^{n-1} i_{s}<\frac{n(n-1)}{2}$, then $0, i_{1}, \cdots, i_{n-1}$ are not all distinct. Therefore $\mathcal{R}(\mathcal{L}, \cdots, \mathcal{L})$ contains $\sum_{\sigma \in \mathbb{S}_{n}} \operatorname{sgn}(\sigma) x_{\sigma(1)} \otimes \cdots \otimes x_{\sigma(n)}$ by Definition 8. Moreover 


$$
\begin{aligned}
& \Phi_{\mathcal{L}_{1}, \cdots, \mathcal{L}_{n}}\left(\sum_{\sigma \in \subseteq_{n}} \operatorname{sgn}(\tau) x_{\tau(1)} \otimes \cdots \otimes x_{\tau(n)}\right) \\
& =\frac{1}{n !} \sum_{\tau \in \mathbb{S}_{n}} \operatorname{sgn}(\tau)\left(\sum_{\sigma \in \subseteq_{n}} \operatorname{sgn}(\sigma) x_{(\sigma \tau(1))}\left(d x_{(\sigma \tau(2))}\right) \cdots\left(d^{n-1} x_{(\sigma \tau(n))}\right)\right) \\
& =\sum_{\sigma \in \subseteq_{n}} \operatorname{sgn}(\sigma) x_{(\sigma(1))}\left(d x_{(\sigma(2)))}\right) \cdots\left(d^{n-1} x_{(\sigma(n))}\right) \\
& =\Phi_{\mathcal{L}}^{(n)}\left(x_{1} \wedge \cdots \wedge x_{n}\right) .
\end{aligned}
$$

This complete the proof.

Q.E.D.

LEMMA 18. The image of $\Phi_{\perp_{1}, \ldots, \mathcal{L}}$ is equal to the image of $\Phi_{\perp}^{(n)}$.

PROOF. Let $\tau=\Sigma x_{j}^{(1)} \otimes \cdots \otimes x_{n}^{(n)}$ be an element of $\mathscr{R}(\mathcal{L}, \cdots, \mathcal{L})$. Then

$$
\begin{aligned}
\Phi_{\mathcal{L}, \ldots, \mathcal{L}}(\tau) & =\frac{1}{n !} \sum_{j}\left(\sum_{\sigma \in \Phi_{n}} \operatorname{sgn}(\sigma) x_{j}^{(\sigma(1))}\left(d x_{j}^{(\sigma(2))}\right) \cdots\left(d^{n-1} x_{j}^{(\sigma(n))}\right)\right. \\
& =\frac{1}{n !} \sum_{j}\left|\begin{array}{ccc}
x_{j}^{(1)} & \cdots & d^{n-1} x_{j}^{(1)} \\
\vdots & & \vdots \\
x_{j}^{(n)} & \cdots & d^{n-1} x_{j}^{(n)}
\end{array}\right| \\
& =\frac{1}{n !} \sum_{j} \Phi_{\mathcal{L}}^{(n)}\left(x_{1} \wedge \cdots \wedge x_{n}\right) .
\end{aligned}
$$

Therefore the image of $\Phi_{\mathcal{L}, \ldots, \mathcal{L}}$ is contained in the image of $\Phi_{\mathcal{L}}^{(n)}$. The converse is clear by Lemma 17 .

Q.E.D.

LEMMA 19. Let $\mathcal{L}_{1}, \cdots, \mathcal{L}_{n}$ and $\mathcal{Q}$ be invertible sheaves on $C$. Then the following diagram is commutative:

$$
\begin{gathered}
\mathscr{R}\left(\mathcal{L}_{1}, \cdots, \mathcal{L}_{i}, \cdots, \mathcal{L}_{n}\right) \otimes \Gamma(C, \mathcal{L}) \\
\Phi_{\mathcal{L}_{1}, \cdots, \mathcal{L}_{n} \otimes i d} \stackrel{\text { cup product }}{\longrightarrow} \\
\Gamma\left(C, \omega_{C}^{\otimes n(n-1) / 2} \otimes \mathcal{L}_{1} \otimes \cdots \otimes \mathcal{L}_{i} \otimes \cdots \otimes \mathcal{L}_{n}\right) \otimes \Gamma(C, \mathscr{P}) \stackrel{\text { cup product }}{\longrightarrow} \\
\mathscr{R}\left(\mathcal{L}_{1}, \cdots, \mathcal{L}_{i} \otimes \mathcal{P}, \cdots, \mathcal{L}_{n}\right) \\
\Phi_{\mathcal{L}_{1}, \cdots, \mathcal{L}_{i} \otimes \mathcal{L}, \cdots, \mathcal{L}_{n} \downarrow} \\
\Gamma\left(C, \omega_{C}^{\otimes n(n-1) / 2} \otimes \mathcal{L}_{1} \otimes \cdots \otimes \mathcal{L}_{i} \otimes \mathscr{P} \otimes \cdots \otimes \mathcal{L}_{n}\right)
\end{gathered}
$$

Proof. This lemma is clear by the same calculations as in Lemma 16.

Q.E.D.

LEMMA 20. Let $\mathcal{L}$ and $\mathscr{M}$ be invertible sheaves on $C$. If $\operatorname{deg}(\mathcal{L}) \geqq 2 g+1$ and $\operatorname{deg}(\mathscr{M}) \geqq$ then $\Gamma(C, \mathcal{L}) \otimes \Gamma(C, \mathscr{M}) \rightarrow \Gamma(C, \mathcal{L} \otimes \mathscr{M})$ is surjective. 
Proof. See Fujita [6] p. 168 Proposition 1, 10, Q.E.D.

Proposition 2. Let $\mathcal{L}$ be an invertible sheaf on $C$. If

$$
\operatorname{deg}(\mathcal{L}) \geqq(g-1)\left(2 n^{2}-2 n+3\right)+2\left(n^{2}-1\right),
$$

then there is an effective divisor $A$ of degree $g+n$ such that $\Gamma(C, \mathcal{O}(A)$ ) is an $(n-1)$-immersive $(n+1)$-net and

$$
H^{1}\left(C,\left(\omega_{C}^{\otimes n^{2-4 n+5}} \otimes \mathcal{O}(A)^{\otimes 2 n-2}\right)^{\otimes-1} \mathcal{L}\right)=0
$$

Proof. If $A$ is a general effective divisor of degree $g+n$, then $\operatorname{dim} \Gamma(C, \mathcal{O}(A-n P))=1$ for every $P \in C$. This implies that $\Gamma(C, \mathcal{O}(A))$ is an $(n-1)$-immersive $(n+1)$-net. Moreover the condition

$$
H^{1}\left(C,\left(\omega_{C}^{\otimes n^{2}-4 n+5} \otimes \mathcal{O}(A)^{\otimes 2 n-2}\right)^{\otimes-1} \otimes \mathcal{L}\right)=0
$$

is also a general condition. Therefore we have the result.

Q.E.D.

Now we prove the Main theorem.

THEOREM 2. Let $\mathcal{L}$ be an invertible sheaf on $C$. If

$$
\operatorname{deg}(\mathcal{L}) \geqq(g-1)\left(2 n^{2}-2 n+3\right)+2\left(n^{2}-1\right),
$$

then the $n$-Wahl map

$$
\Phi_{\mathcal{L}}^{(n)}: \wedge^{n} \Gamma(C, \mathcal{L}) \longrightarrow \Gamma\left(C, \omega_{C}^{\otimes n(n-1) / 2} \otimes \mathcal{L}^{\otimes n}\right)
$$

is surjective.

Proof. Let $A$ be an effective divisor in Proposition 2. By Lemma 18, Lemma 19 and Theorem 1, $\Phi_{\mathcal{O}(A), \ldots O(A), O(A), \mathcal{L}}$ is surjective. As

$$
\operatorname{deg}(\mathcal{L} \otimes \mathcal{O}(A)) \geqq 2 g \text { and } \operatorname{deg}\left(\omega_{\mathcal{C}^{n(n-1) / 2}} \otimes \mathcal{O}(A)^{\otimes n-1} \otimes \mathcal{L}\right) \geqq 2 g+1,
$$

a cup product map

$$
\begin{gathered}
\mu: \Gamma\left(C, \omega_{C}^{\otimes n(n-1) / 2} \otimes \mathcal{O}(A)^{\otimes n-1} \otimes \mathcal{L}\right) \otimes \Gamma(C, \mathcal{L} \otimes \mathcal{O}(-A))^{\otimes n-1} \\
\longrightarrow \Gamma\left(C, \omega_{c}^{\otimes n(n-1) / 2} \otimes \mathcal{L}^{\otimes n}\right)
\end{gathered}
$$

is surjective by Lemma 20, By Lemma 19 we have the following commutative diagram:

$$
\begin{array}{cc}
\Gamma\left(C, \omega_{C}^{\otimes n(n-1) / 2} \otimes \mathcal{O}(A)^{\otimes n-1} \otimes \mathcal{L}\right) \otimes \Gamma(C, \mathcal{L} \otimes \mathcal{O}(-A))^{\otimes n-1} \stackrel{\mu}{\longrightarrow} \Gamma\left(C, \omega_{D}^{\otimes n(n-1) / 2} \otimes \mathcal{L}^{\otimes)}\right. \\
\Phi_{\mathcal{O}(A), \ldots \mathcal{O}(A), \mathcal{O}(A), \mathcal{L} \uparrow} \Phi_{\mathcal{L}, \cdots, \mathcal{L} \uparrow} \uparrow \\
\mathcal{R}(\mathcal{O}(A), \cdots, \mathcal{O}(A), \mathcal{L}) \otimes \Gamma(C, \mathcal{L} \otimes \mathcal{O}(-A))^{\otimes n-1} \longrightarrow & \mathcal{R}(\mathcal{L}, \cdots, \mathcal{L}) .
\end{array}
$$


As $\mu$ and $\Phi_{\mathcal{O}(\boldsymbol{A}), \ldots(\mathcal{O}), \mathcal{O}(\boldsymbol{A}), \mathcal{L}}$ are surjective, we have that $\Phi_{\mathcal{L} \ldots, \mathcal{L}}$ is surjective. Therefore $\Phi_{\mathcal{L}}^{(n)}$ is surjective by Lemma 18. Q.E.D.

\title{
References
}

[1] E. Arbarello, M. Cornalba, P. A. Griffiths and J. Harris, Geometry of Algebraic curves 1, Springer-Verlag, 1985.

[2] J.N. Brauner, The Gaussian map $\Phi_{K}$ for curves with special linear series, Thesis University of North Carolina, 1992.

[3] C. Cilberto, J. Harris and R. Miranda, On the surjectivity of the Wahl map, Duke Math., 57 (1988), 829-858.

[4] C. Cilberto and R. Miranda, On the Gaussian map for canonical curves of low genus, Duke Math., 81 (1990), 417-443.

[5] H. M. Farkas and I. Kra, Riemann surfaces, Springer-Verlag, 1980.

[6] T. Fujita, Defining equations for certain types of polarized varieties, Complex Analysis and Algebraic Geometry, Iwanami, Tokyo, 1977.

] 7 A. Garcia and J.F. Voloch, Duality for projective curves, Bol. Soc. Bras. Mat. (N.S.), 21 (1991), 159-175.

[8] M. Green, Koszul cohomology and the geometry of projective varieties, J. Differential Geometry, 19 (1984), 125-171.

[9] R. Hartshorne, Algebraic Geometry, Springer-Verlag, 1974.

[10] A. Hefetz and S. Kleiman, Notes on the duality of projective varieties, Geometry today, Int. Conf., Roma 1984, Prog. Math., 80 (1985), 143-183.

[11] F. Hirzeburch, Topological methods in Algebraic Geometry, Springer-Verlag, 1966.

[12] S. Iitaka, Algebraic Geometry, Springer-Verlag, 1982.

[13] D. Laksov, Wronskians and Plücker formulas for linear systems on curves, Ann. scient. Ec. Norm. Sup, 17 (1984), 17-45.

[14] D. Mumford, Varieties defined by quadratic equations, Questioni sulle varieta algebiche, Corsi dal C. I. M.E., Editoni Cremonese, Roma, (1969), 29-100.

[15] A. Ohbuchi, On the projective normality of some varieties of degree 5, Pacific Journal of Math., 144 (1990), 313-325.

[16] J. Wahl, The Jacobian algebra of a graded Gorenstein singularity, Duke Math. J., 55 (1987), 843-871.

[17] J. Wahl, Deformations of quasi-homogeneous singularities, Math. Ann., 280 (1988), 105-128.

[18] J. Wahl, Gaussian maps on algebraic curves, J. Differential Geometry, 32 (1990), 77-98.

\author{
Department of Mathematics \\ Faculty of Education \\ Yamaguchi University \\ Yamaguchi, 753 \\ Japan
}

E-mail address: akira@ccyi.ccy.yamaguti-u.ac.jp 